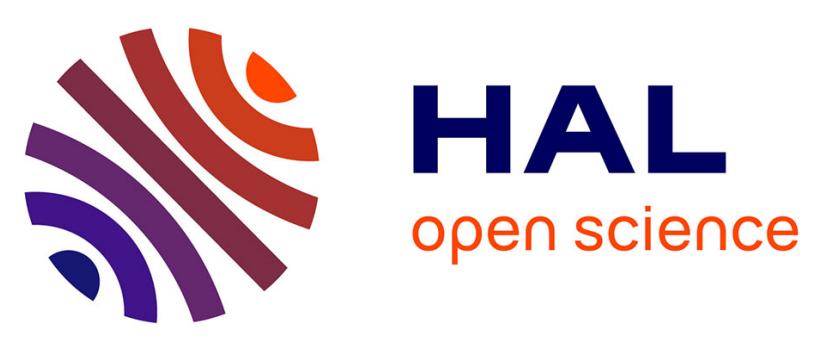

\title{
Assessment of the detection abilities of monitoring networks for passive tracers at local and regional scales
}

\author{
I. Korsakissok, Vivien Mallet, Bruno Sportisse
}

\section{To cite this version:}

I. Korsakissok, Vivien Mallet, Bruno Sportisse. Assessment of the detection abilities of monitoring networks for passive tracers at local and regional scales. Atmospheric Environment, 2010, 44 (3), pp.408-420. 10.1016/j.atmosenv.2009.10.022 . inria-00543191

\section{HAL Id: inria-00543191 \\ https://hal.inria.fr/inria-00543191}

Submitted on 6 Dec 2010

HAL is a multi-disciplinary open access archive for the deposit and dissemination of scientific research documents, whether they are published or not. The documents may come from teaching and research institutions in France or abroad, or from public or private research centers.
L'archive ouverte pluridisciplinaire HAL, est destinée au dépôt et à la diffusion de documents scientifiques de niveau recherche, publiés ou non, émanant des établissements d'enseignement et de recherche français ou étrangers, des laboratoires publics ou privés. 


\title{
Assessment of the detection abilities of monitoring networks for passive tracers at local and regional scales
}

\author{
Irène Korsakissok ${ }^{*, a, b}$, Vivien Mallet ${ }^{\mathrm{b}, \mathrm{a}}$, Bruno Sportisse $^{\mathrm{b}, \mathrm{a}}$ \\ ${ }^{a}$ CEREA, Joint Research Laboratory, ENPC / EDF REDD, Université Paris Est, 6-8 avenue Blaise Pascal, Cité \\ Descartes, 77455 Champs-sur-Marne, Marne la Vallée Cedex 2 , France \\ ${ }^{b}$ INRIA, Paris-Rocquencourt Research Center, B.P. 105, 78153 Le Chesnay Cedex, France
}

\begin{abstract}
We propose a method to evaluate the detection abilities of networks used for protection purposes. Such networks are designed for the detection of nuclear, biological or gaseous emissions, without constraint on the source location. Their assigned goal is to have the best chance to detect a threatening emission located anywhere in the vicinity of a domain to protect. Two sensors siting applications are addressed: sensors placed in the surroundings of a facility to protect, or sensors carried by people scattered within a small area. A network protection ability is related both to its detection scope, and to its response time. To assess the performance of such networks, two statistical indicators are therefore designed: the detection probability, computed on a large number of possible source locations, and the saturation time, which is the time when the maximum detection probability has been reached.

Simulations are then carried out with the Polyphemus air quality modeling system for many emission scenarios, including 961 possible source locations, various emitted species, and a few representative meteorological situations. This allows to assess the performance of single sensors as well as full networks, and their sensitivity to parameters like meteorological conditions and source characteristics. The emitted quantity and meteorological dispersion are found to be important parameters, whereas the species type does not significantly influence the results. Two network design methods are considered: (1) networks composed of a given number of the "best" sensors according to an indicator, and (2) sensors placed in circles around the protected domain. The networks built with respect to the detection probability show good results with a limited number of sensors, while the saturation time is not reliable enough to build networks. The networks based on circles also show a good performance in the studied cases, provided there is a sufficient number of sensors.
\end{abstract}

Key words: Detection network, passive tracer, Polyphemus, local scale, regional scale

\section{Introduction}

\subsection{Context and objectives}

The design, evaluation and optimization of monitoring networks have already been investigated, both in the field of atmospheric chemistry for ozone and its precursors ([9], [4]), and to

${ }^{*}$ Corresponding author. E-mail: korsakissok@ cerea.enpc.fr Tel: +33 (0)164152168 Fax: +33(0)1641521 70

Preprint submitted to Elsevier

November 27, 2010 
monitor accidental releases of radionuclides. Studies on the latter subject rely on the assumption of a few well-known possible source locations, corresponding to power plant locations. They have been carried out at several spatial scales, from local to global: near-field studies involve measurement sensors placed within $30 \mathrm{~km}$ of nuclear power plants, whether at fixed locations [7] or with mobile device [5], while the optimization of monitoring networks at mesoscale (up to $2000 \mathrm{~km}$ ) is investigated in [1]. The monitoring of radionuclides for military applications at global scale is thoroughly assessed by the CTBT organization ("Comprehensive nuclear TestBan Treaty", [10]). All these applications share two main purposes: the detection (of an accident or a pollution peak), and the reconstruction of the concentration fields on the basis of the measurements. Most studies focus on the networks ability to reconstruct the map of concentrations, which involves indicators such as the difference between the reconstructed concentrations and the true field (given by simulations, or measurements when available).

This paper addresses a similar issue, but in a military or homeland security context: a delimited area-a facility, a high-profile event, a combat unit, or dignitaries-has to be protected from any hazardous air pollutant. The pollutant may be released with harmful intent from a single ground location in the vicinity of the domain, and the source location and characteristics are unknown. Two sensor siting applications are addressed. In the first case, fixed sensors may be placed around a zone containing buildings to be protected. In the second case, people carrying detection devices are scattered within an area containing small units. In both cases, the protection ability of a network is determined by its ability to detect a release, wherever its location. In addition, a performance criterion related to the response time can be defined, in cases an advanced warning is needed (for instance, if the released species quickly reaches harmful concentrations with immediate health effects). Therefore, appropriate performance indicators should be designed, to quantify the network abilities in both respects. The aim of this study is to explore the new framework with (1) the design of performance indicators for such networks, (2) relevant tests in different meteorological and emission scenarios, and (3) the design of several networks based on the performance indicators. The chosen test scenarios are highly idealized (perfect sensors, simple domain shapes...) and should be adapted to real-life situations. The underlying objective is to issue general recommendations regarding the performance indicators and the methods to be retained in the design of a detection network for real cases.

\subsection{Methodology and outline}

In this study, two sets of simulations are performed: the first one corresponds to the buildings protection scenario, with a $50 \times 50 \mathrm{~km}^{2}$ domain, and the second one is the small-units protection scenario, with a $10 \times 10 \mathrm{~km}^{2}$ domain. The simulations are carried out with the air quality modeling system Polyphemus [8], using an Eulerian model in the former case and a Gaussian model in the latter. For both scenarios, many simulations are performed (1) to account for many sources locations and (2) to include several typical meteorological situations. This generates a large database from which detection capabilities are assessed. The simulation scenarios are described in Section 2. Two statistical indicators are used to quantify the detection abilities: the detection probability and the saturation time-see Section 3. The simulation database allows one to compute these indicators for any possible sensor location (Section 4) or network configuration (Section 5). For both sensors and networks, Sections 4 and 5 address the sensitivity of the indicators to parameters like the meteorological conditions or the source characteristics. In Section 5, two families of networks are considered. In the first one, the networks are composed of the best sensors according to the detection probability. In the second family, the networks are designed 
with concentric circles. This particular geometry is chosen because of its simple design, and it could provide good results without further optimization.

\section{Test scenarios}

\subsection{Domains and computing strategy}

In our context, a delimited area (the "protected domain" $D_{p}$ ) has to be protected from an hazardous air pollutant. A dangerous source is defined as a source that can contaminate the domain $D_{p}$, that is, triggers above-threshold concentrations in at least one point of $D_{p}$. This is one of the hypothesis made in this study, where idealized cases are presented. The main assumptions are:

1. Immediate contamination of the protected domain, as soon as one sensor within $D_{p}$ detects the pollutant,

2. Square-shaped domains, while a real-case study might account for more complex shapes (oblong or L-shaped buildings) and land-use coverage (e.g., water might forbid siting sensors at some locations),

3. Perfect sensors, with immediate response, and a low detection threshold (see Section 3.1).

In addition to the protected area, several domains are defined (see Figure 1):

- $D_{c}$ is the area where sensors may be placed (one per cell). It includes $D_{p}$. Some sensors can be placed within $D_{p}$.

- $D_{s}$ contains all dangerous sources (one per cell). It includes $D_{c}$.

- $D_{M}$ is the modeled domain. It includes all other domains.

In this section, the size of these domains is detailed, along with the simulation set-up, for the two base test scenarios: the buildings protection scenario ("regional scale"), and the small-units protection scenario ("local scale").

\subsubsection{Buildings protection scenario}

Here, the domain $D_{p}$ is a facility (military base, for example). The sensors are placed at fixed locations, either within the base or in its surroundings. The main purpose in this case is advanced warning, so the alert must be raised before the pollutant reaches $D_{p}$. This early-warning criterion could be relaxed, for example in the case of a biological attack, where medicine could be given some time after the event. The simulations are carried out with the Eulerian model Polair3D [3]. The domains $D_{p}, D_{c}, D_{s}$ and $D_{M}$ are defined in Table 1. The simulation grid has a resolution of $0.01^{\circ}$ in the horizontal, which is about $1 \mathrm{~km}$. It includes ten vertical layers, up to $3060 \mathrm{~m}$ above ground. The height of the first layer is $30 \mathrm{~m}$. The numerical time step is $60 \mathrm{~s}$, and a simulation covers a time period of 3 hours. There are $50 \times 50$ cells in $D_{M}$, and meteorological conditions as well as land use can differ from one cell to another (although the terrain is flat and rural). 


\subsubsection{Small-units protection scenario}

In this scenario $D_{p}$ is a zone containing people (military units, dignitaries...), and the sensors are supposed to be scattered among them (typically, people carrying detection devices) so that $D_{c}=D_{p}$. Therefore, there is no advanced warning here, and it is assumed that the sensors threshold is lower than the minimum harmful concentrations, and that a rapid response is not a critical issue. The domains are defined in Table 2. The simulations are carried out with the Gaussian puff model of the Polyphemus platform [6]. Contrary to the Eulerian model, the Gaussian model gives an analytical formula to compute the concentrations, and does not need a grid. The grid is used in order to consider a finite number of source and sensor locations. The concentrations are computed in each cell of the gridded domain $D_{c}$, at ground level only. The simulation duration is 3 hours, the time step is $100 \mathrm{~s}$, and the space step in the horizontal is $200 \mathrm{~m}$. Here, the meteorological data and the land type are supposed to be homogeneous.

\subsection{Sources}

The simulations are carried out for three species, the first with radioactive decay, the second with biological decay and the third with no decay at all (simply passive). The timescale of the decay is of the order of 8 days for the radioactive species, gaseous iodine, which makes it almost passive. The biological species decays with a characteristic time of ten minutes (during daytime) or twenty minutes (during nighttime). It is modeled as particulate matter (PM) with diameters of $0.5 \mu \mathrm{m}, 5 \mu \mathrm{m}$ and $10 \mu \mathrm{m}$. The third species is a purely passive gaseous species (GM for gaseous matter). Table 3 gives the emitted quantities. Wet scavenging and dry deposition are taken into account for the three species. The dry-deposition velocity of the two gaseous species is supposed to be constant and equal to $5.10^{-3} \mathrm{~m} \mathrm{~s}^{-1}$. The Belot parameterization [2] is used to estimate their scavenging coefficients. For particulate matter, the dry-deposition velocities are computed for the several diameters (with gravitational settling), and the scavenging coefficients are computed according to the Slinn parameterization [11].

\subsection{Meteorological scenarios}

Because of the large number of simulations (one per possible source), running simulations for a full year is too high a computational burden. Therefore, we focused on a few typical meteorological situations. Low-dispersion cases were privileged, since high concentrations were supposed to be the most critical from a health response standpoint. Thus, the selected situations do not account for all possible wind directions. However, changing the source location is more or less equivalent to changing the wind direction, at least for a circular geometry (in the case of square domains, some "corner effects" can appear, see Section 5.1 for instance). In addition, one can assume that the wind direction is known in the two kinds of sensors siting applications addressed here:

- Protecting a building: the most frequent wind direction(s) are well known and can be taken into account in a prospective study to place sensors,

- Protecting small units: there is no need to cover a wide range of meteorological situations since the sensors are placed in real-time.

Data for the Eulerian model were provided by a preliminary study of ECMWF meteorological data for year 2004 in the French region of Bourges (chosen because of its flat terrain). Four situations were retained. The first two cases, hereafter called "case" or "situation" 0 and 1 respectively, are winter cases of low dispersion. The third case, labelled "situation 2", is a summer case 
with very low dispersion during night, and substantial rain. The last case is a summer situation of medium dispersion. Table 4 gives the averaged values of the wind speed, the boundary layer height, the rain, the cloudiness and the Pasquill stability class, for the four situations. Figure 2 shows the maps of wind directions for the four meteorological situations at the beginning of the simulation (emission time). The maps give a good idea of the general wind direction, except for case 2 where the wind turns during the simulation, and partly comes from the south at the end of the three hours.

In the Gaussian model, the meteorological data is assumed to be homogeneous and constant. Therefore, these are synthetic cases, designed to share the main dispersion characteristics (low or medium dispersion, rain) with the regional scale. The wind speed, the boundary layer height, the rain, the Pasquill stability class are taken in one cell of the regional domain, and summarized Table 5. Only the wind directions have been shifted and therefore differ from the regional values (Figure 3), in order to account for various wind directions.

\subsection{Simulations}

The simulations are carried out for all sources in the domain $D_{s}$, which in both test scenarios amounts to 961 sources: the regional domain $D_{s}$ covers $31 \times 31 \mathrm{~km}^{2}$ and the discretization is $1 \mathrm{~km}$, and the local domain is $6.2 \times 6.2 \mathrm{~km}^{2}$ wide, with cells of size $200 \mathrm{~m}$. There are four meteorological situations, two gaseous species and three particulate matter diameters, which corresponds to $20 \times 961$ simulations in total. Once the simulations are gathered into a "concentration database", one may compute the performance indicators for any network, any species and any meteorological situation. In Section 5.3, additional simulations are carried out with variations of wind speed and wind angle, on the basis of meteorological situation 1 and for one species (iodine). There are 8 variations of wind speed and 12 variations of wind angle, so the number of extra runs required for the sensitivity study amounts to $20 \times 961$.

\section{Sensor and network statistics}

\subsection{Statistical indicators for sensors}

A sensor is defined by a position, the species it monitors, a detection threshold $C_{\min }$ (minimum concentration for the species to be detected), and a minimum duration $t_{\min }$. A species is detected if and only if its concentration remains above the detection threshold $C_{\min }$ for at least $t_{\min }$. In this study, $C_{\text {min }}=0.01 \mu \mathrm{g} \mathrm{m}^{-3}$ and $t_{\min }$ is only limited by the simulation time step $\Delta t$. A sensor may be placed at the center of any cell in the domain $D_{c}$. Let $k$ be the index of that cell for a given sensor. For this sensor $k$, we define the following indicators:

1. The arrival time of a given source $s, t_{a r r}^{k}(s)$ :

$$
t_{\text {arr }}^{k}(s)=\min \left\{t \mid C(t)>C_{\min } \text { and } d(t) \geq t_{\min }\right\},
$$

with $C(t)$ the concentration at the sensor location and at time $t$ for the species emitted by the source $s$, and $d(t)$ the time during which $C(t)$ has been above the sensor threshold $C_{\min }$. Thus, $t_{a r r}^{k}(s)$ is the first date at which the source is detected by the sensor. It is the sum of the propagation time from the source to the sensor, and of $t_{\min }$. It is set to zero if the source $s$ is never detected by the sensor. 
2. The sensor detection probability at time $t, P^{k}(t)$. It is the proportion of sources that have been detected by sensor $k$ at time $t$.

$$
P^{k}(t)=\frac{1}{N_{s}} \sum_{s=0}^{N_{s}-1} P_{s}^{k}(t)
$$

where $P_{s}^{k}(t)$ is equal to zero if the source $s$ has not been detected by the sensor at time $t$ and 1 otherwise, that is:

$$
P_{s}^{k}(t)= \begin{cases}1 & \text { if } t_{a r r}^{k} \neq 0 \text { and } t \geq t_{\text {arr }}^{k}, \\ 0 & \text { otherwise. }\end{cases}
$$

In Equation 2, $N_{s}$ is the number of sources, among all possible sources within $D_{s}$, that actually reach $D_{p}$ (so-called effective sources). Thus, all sources downwind of $D_{p}$ are ignored, which prevents a needless increase in $N_{s}$ and an artificial decrease of the detection probabilities.

In order to quickly assess a sensor performance, it is useful to define more global indicators, that do not depend on time or source. In this study, we focus on the maximum detection probability $P_{\max }^{k}$ of a sensor $k$ :

$$
P_{\text {max }}^{k}=\max _{t} P^{k}(t)=P^{k}(+\infty) .
$$

In addition, we also define the saturation time $t_{\text {sat }}^{k}$, which is the time when the maximum detection probability of sensor $k$ has been reached:

$$
t_{\text {sat }}^{k}=\inf \left\{t \mid P^{k}(t)=P_{\text {max }}^{k}\right\} .
$$

Note that all indicators involving the arrival time are dependent on the simulation time step: the finer the time step, the better the discrimination between two sensors based on time constraints.

\subsection{Constraints on the sensors}

In the buildings protection scenario, there is an additional condition: the source must be detected before it contaminates $D_{p}$ (this is irrelevant at local scale where $D_{p}=D_{c}$ ). Thus, we define $t_{d p}$ as the time when the source first reaches $D_{p}$ :

$$
t_{d p}=\inf \left\{t_{a r r}^{k} \mid k \in D_{p}\right\}
$$

In that case, Equation 3 is modified as follows:

$$
P_{s}^{k}(t)= \begin{cases}1 & \text { if } 0<t_{a r r}^{k} \leq t_{d p} \text { and } t \geq t_{a r r}^{k} . \\ 0 & \text { otherwise. }\end{cases}
$$

This criterion could be modified, depending on the desired time interval between the warning and the contamination of $D_{p}$. In the following, a detection at time $t_{d p}$ is considered successful, so sensors located within $D_{p}$ can have a non-zero detection probability. 


\subsection{Statistical indicators for networks}

A network is defined as a set of sensors. Its detection probability and arrival time can be defined similarly to the case of a single sensor:

$$
P^{\text {network }}(t)=\frac{1}{N_{s}} \sum_{s=0}^{N_{s}-1} P_{s}^{\text {network }}(t),
$$

where $P_{s}^{\text {network }}(t)$ is equal to 0 if the source $s$ has not been detected by the network and 1 otherwise. As soon as $P_{s}^{k}(t)=1$ for a given sensor $k$ of the network, $P_{s}{ }^{\text {network }}(t)=1$. It follows that

$$
P_{s}{ }^{\text {network }}(t)=\max _{k} P_{s}^{k}(t),
$$

where $k$ is in $\llbracket 0, N_{\text {sensor }} \llbracket$ if $N_{\text {sensor }}$ is the number of sensors in the network.

The arrival time of the network for the source $s$ is:

$$
t_{\text {arr }}^{\text {network }}(s)=\min _{k} t_{\text {arr }}^{k}(s) .
$$

Finally, the maximum detection probability and saturation time of a network are

$$
P_{\max }^{\text {network }}=\max _{t} P^{\text {network }}(t)=P^{\text {network }}(+\infty)
$$

and

$$
t_{\text {sat }}^{\text {network }}=\inf \left\{t \mid P^{\text {network }}(t)=P_{\max }^{\text {network }}\right\} .
$$

\section{Sensors performance}

Before defining any network, it is useful to have a glimpse of the performance of all individual sensors within the domain $D_{c}$ : any network is a subset of this ensemble of sensors. In particular, the networks considered in Section 5.1 are composed of the best sensors (according to a given indicator), whose positions can be inferred from the maps of detection probability $P_{\max }$ and saturation time $t_{\text {sat }}$. The maps also give insights on the sensors sensitivity to several parameters such as the emitted quantity, the dispersion, and the rain. They allow to address the following questions: to what extent do the conclusions of the study depend on the simulation scenarios? How to choose the best scenarios in order to be representative of the networks general performance?

\subsection{Influence of scenarios and meteorology}

Only $D_{c}$ is shown on the maps, and the borders of $D_{p}$ are indicated when necessary. Figure 4 shows the maps of detection probabilities for iodine and all meteorological cases for the buildings protection scenario, and Figure 5 for the small-units protection scenario. In the former case, the Eulerian simulations are carried out with non-homegenous conditions (meteorology and land-use), while the latter case corresponds to the Gaussian simulations, with constant and homogeneous conditions. The sensors performance depends, quite obviously, on the wind direction. While this is easily verified in the Gaussian results (Figure 5), the "main wind direction" is not so clear with the Eulerian model, due to the inhomogeneous and unstationnary conditions (e.g., the wind turns from the west-north west to south direction in the case 2). Meanwhile, the other 
characteristics of the dispersion (wind speed and turbulence strength) may have more complex effects on the results. The effects of a very low-dispersion case like situation 2 are twofold: (1) higher concentrations, and (2) lower horizontal spread of the emitted plume. The former has consequences similar to a small increase of the emitted quantity: there are more effective sources, and $P_{\max }$ is higher at the downwind part of the domain, which results in a more homogeneous map of detection probabilities. Conversely, the medium-dispersion case (situation 3) results in higher discrepancies between the sensors. On the other hand, a lower plume spread leads to a decrease in the number of effective sources as well as in detection probabilities (a narrower plume reaches fewer sensors).

The main differences between the two scenarios are due to the additional constraint in the buildings protection case (Section 3.2): a sensor successfully detects a source only if it does so before the pollutant reaches $D_{p}$. Thus, sensors within $D_{p}$ show a very low detection probability, and the best sensors according to $P_{\max }$ are located near the boundary of $D_{p}$ (more or less upwind). On the contrary, in the units protection scenario, $D_{c}=D_{p}$, and the sensors with the highest detection probability are placed at the farthest end of the domain, downwind. The maps of $t_{\text {sat }}$, not shown here, are exactly the "reverse" of the $P_{\max }$ maps in this case: the farthest sensors also have the highest saturation times. This suggests that coupling the two indicators could lead to a good compromise, if a time criterion had to be applied in this scenario.

\subsection{Emitted quantities and loss processes}

The maps of $P_{\max }$ and $t_{s a t}$ at regional scale are shown in Figure 6 for the meteorological situation 1 and all species. It shows significant differences between the emitted species. There are two possible reasons for these differences: (1) the emitted quantities and (2) the loss processes. In meteorological situation 1, there is no wet scavenging: the only loss processes are dry deposition and linear decay. Since the two gaseous species have the same deposition velocities, and the timescale of radioactive decay for iodine is several days, the differences between the maps for GM and iodine are due to the emitted quantity ( $1 \mathrm{~kg}$ for iodine and $5 \mathrm{~kg}$ for GM, see Table 3 ). The biological species has the highest emitted quantity $(10 \mathrm{~kg})$, but it undergoes a significant biological decay. Since all the processes involved in the dispersion are linear, the emitted quantity would not be an important parameter but for the sensors threshold. Increasing the quantity emitted by one source induces higher concentrations: the plume stays longer above the threshold, and can be detected by sensors at farther distances (downwind or crosswind). Thus, the maps of $P_{\max }$ become more homogeneous, since a source is detected by almost all sensors, even the farthest downwind (Figures 6(c) and 6(e)). This also leads to higher saturation times, since farther sources are detected. This raises the issue of the number of effective sources (as defined in Section 3), which obviously increases with the emitted quantity. The size of $D_{s}$ should therefore be modified accordingly, to account for all possible sources. Otherwise, the maps are not representative of the real sensors performance, and sensors placed downwind of $D_{p}$ show an unrealistic detection probability. This condition is verified for the situation 1 and iodine, but not for all quantities tested here. In the rest of the paper, the reference species used is therefore iodine.

Figure 7 allows to assess the influence of loss processes (wet scavenging and dry deposition) for the same emitted quantity: the maps of detection probability are shown for the biological species and three diameters, for the meteorological situation 2 (with rain). The detection probabilities of the coarsest particles are slightly lower, due to the strong scavenging, but there are no significant differences otherwise. 


\section{Networks performance}

In this section the performance of two types of networks is evaluated:

1. Networks composed of the sensors with the highest detection probabilities,

2. Networks defined with a geometry of concentric circles.

These networks are studied at local and regional scale, for all meteorological situations and for iodine. The results for networks composed of the sensors having the best saturation times are not shown, since they give an overall poor performance, except for an excessively large number of sensors (over 200). This indicator is still, however, used to evaluate the networks behavior with respect to time.

\subsection{Networks defined with respect to detection probability}

The networks evaluated in this section comprise the $N_{\text {sensor }}$ sensors having the highest detection probabilities (see Figure 4 and Figure 5 for the sensors positions with $N_{\text {sensor }}=30$ ). For 13 networks with $5 \leq N_{\text {sensor }} \leq 100$, the statistics described in Section 3.3 are computed for all meteorological situations.

Figure 8 shows the networks performance against the number of sensors, for the four meteorological cases. The high detection probability of networks comprising a few sensors suggests a good complementarity between the sensors (they do not detect the same sources), which is a nontrivial fact considering the small sensors spacing. In the units protection scenario, however, this complementarity is mitigated in cases when all the best sensors are located in one corner of the domain $D_{c}$ (cases 1 and 3-see Figure 5). This "corner effect" is avoided in the buildings protection scenario, since all sensors are placed around $D_{p}$ - due to the time constraints-rather than at the border of $D_{c}$. The detection probabilities are not very sensitive to the meteorological situation. $P_{\max }$ for situation 2 is very high in both scenarios, despite the low detection probabilities of individual sensors highlighted in Section 4, especially at local scale. This may be related to the narrow horizontal plume spread in this meteorological situation: each sensor detects few sources, but it does not detect the same sources as its neighbors. In this situation, $P_{\max }$ therefore increases very rapidly with the number of sensors in the network.

In terms of saturation times, Figure 8 shows the good performance of networks at regional scale, compared to the values of $t_{\text {sat }}$ for single sensors. This is consistent with the different patterns of the maps of detection probabilities and saturation times (Figure 6): the sensors with good detection probabilities are not the same as the sensors having the highest saturation times. When increasing the number of sensors in the network, sensors with lower saturation times are rapidly included, contrary to the local-scale scenario which shows scarcely any improvement in $t_{\text {sat }}$ as the number of sensors in the network increases. One may also notice significant differences between the networks saturation times in both scenarios-compared to regional scale, the values of $t_{s a t}$ at local scale highly depend on the situation and are much higher. This probably comes from a slower horizontal diffusion in the Gaussian model than in the Eulerian model.

The overall performance of such networks in terms of detection probability is quite good for all meteorological situations. The saturation times are relatively good (in comparison to single sensors) at regional scale, but this indicator depends too much on the situation to be reliable by itself. These results suggest that a practical way to design a detection network would be to choose a required minimum detection probability, and to adjust the number of sensors in the network in order to decrease the saturation time if necessary. The resulting network should then be tested for several dispersion conditions, and compared to the "best" networks for each situation, as done in 
Section 5.3 where the robustness of such networks with respect to meteorological variations is assessed.

\subsection{Networks defined as concentric circles}

In the buildings protection scenario, one or more concentric circles are placed around $D_{p}$, with a constant distance between two circles. The first circle (internal circle) has a radius $R_{\text {min }}$, and the last one (external circle) has a radius $R_{\max }$. The total number of circles is denoted $N_{\text {circle }}$. Either the number of sensors per circle $N_{\text {sensor }}^{0}$ is constant, or, alternatively, the distance between two sensors $l_{\text {sensor }}$ is fixed (then, the number of sensors increases with the circle radius). In the former case, the total number of sensors in the network is

$$
N_{\text {sensor }}=N_{\text {sensor }}^{0} \times N_{\text {circle }} .
$$

In the latter, the number of sensors is

$$
N_{\text {sensor }}=\sum_{i=1}^{N_{\text {circle }}} 2 \pi R_{i} / l_{\text {sensor }},
$$

where $R_{i}$ is the radius of the circle $i: R_{i}=R_{\min }$ if $i=1$ and $R_{i}=R_{\max }$ if $i=N_{\text {circle. For }}$ example, figure 9 shows two possible configurations (for 3 circles), one with a given $N_{\text {sensor }}^{0}$ and one with a given $l_{\text {sensor }}$. To assess the performance and the robustness of such networks, we study the sensitivity to variations of three parameters: the number of circles, the number of sensors per circle, and the distance between two sensors. The circles are of radii comprised between $R_{\text {min }}=2.5 \mathrm{~km}$ and $R_{\max }=9 \mathrm{~km}$. The circles center is the center of $D_{p}$. Figure 10 shows the results for the following variations:

- Figures 10(a) and 10(b): the number of circles $N_{\text {circle }}$ varies between 1 and 9 circles, with a number of sensors per circle equal to $N_{\text {sensor }}^{0}=5$,

- Figures 10(c) and 10(d): the number of sensors per circle $N_{\text {sensor }}^{0}$ varies between 1 and 14 sensors, with a number of circles equal to $N_{\text {circle }}=3$,

- Figures 10(e) and 10(f): the distance between two sensors $l_{\text {sensor }}$ varies betweent $2 \mathrm{~km}$ and $14 \mathrm{~km}$, with a number of circles equal to $N_{\text {circle }}=3$.

The evolution of $P_{\max }$ and $t_{s a t}$ is represented against the total number of sensors in the network, given by Equation 12 in the first two cases, and by Equation 13 otherwise. This allows to compare the performance in several configurations for a given number of sensors. It can hereby be observed that the detection probabilities reach $100 \%$ in all the configurations for 10 sensors or more. The detection probabilities also are very insensitive to the meteorological situation. Although the saturation times are more sensitive to the meteorology, the variation is very small with 12 sensors or more, which demonstrates a better robustness than the networks studied in Section 5.1. The results are not sensitive to $N_{\text {circle }}$, as long as there are at least two circles (Figures 10(a) and 10(b)), and a large enough number of sensors per circle (here, 5 sensors). Indeed, the networks performance is much lower with less than 4 sensors per circle (Figures 10(c) and 10(d)). With more than 4 sensors, one may have at least one sensor placed in each direction, preventing the dependence to the wind direction, and allowing a sufficient scatter of the sensors. Placing the sensors at constant intervals induces slightly better performance (Figures 10(e) and 10(f)). 
Whatever constraint may be put on the circles, the sensors are scattered throughout the domain and they are therefore very complementary, which enhances the network efficiency. However, it is noteworthy that the performance is less dependent on the actual constraints on the circles than on the minimum number of sensors. One conclusion is that this method is efficient if enough sensors are available - otherwise, it is advocated to place the sensors according to their individual detection probabilities, like in Section 5.1. Another conclusion is that other construction schemes, based on geometric constraints, may be efficient. For instance, simply enforcing a minimum distance between the sensors, without any shape constraint, could be an efficient scheme, combined with a selection based on the detection probability. While considering the inclusion of a new sensor in the network, the detection probability may also be redefined so as to account only for the sources not detected by the network (i.e., the sources already detected would be discarded in Equation 7).

\subsection{Networks sensitivity to wind variations}

The effect of small variations in wind speed and direction is studied, to determine the networks sensitivity to meteorological measurement errors or to small variations in time. For that purpose, meteorological case 1 is taken as a reference, and we study variations of wind speed $v$ between $-35 \%$ and $+35 \%$, with a $5 \%$ step, which should account for measurement errors. In the same way, the wind angle varies from $-25^{\circ}$ to $+25^{\circ}$ with a $5^{\circ}$ step. The networks studied in Section 5.1 for situation 1 are evaluated for each new wind speed or angle variation. The networks defined in Section 5.2 would of course be insensitive to angle variations, although their performance could be affected by changes in the wind speed. Figure 11 shows the influence of wind speed variations at local and regional scale. The mean (of $P_{\text {max }}$ or $t_{\text {sat }}$ ) on all meteorological situations is shown, along with the range of variations (minimum and maximum values on all perturbed simulations). At both scales, the detection probability $P_{\max }$ is scarcely influenced by the wind speed variations. At local scale, $t_{\text {sat }}$ increases by $40 \%$ when the wind speed decreases by $35 \%$, for $N_{\text {sensor }}=10$. The saturation time is, once again, the more sensitive parameter, especially at local scale. Figure 12 shows that variations in the wind angle has similar effects. However, $P_{\max }$ is also affected by the variations at local scale: it may decrease by up to $10 \%$. This sensitivity study is typical of the evaluation to be carried out in practical cases for a given network configuration and a given scenario. It shows the robustness of the networks to wind variations, especially in terms of detection probability, even when they are not placed in concentric circles.

\section{Conclusions}

We investigated the use of monitoring networks for protection purposes, in a military or homeland security context, without constraint on the source location. Two scenarios were addressed, a buildings protection scenario and a small-units protection scenario. Indicators aimed at representing the protection ability of such networks, accounting for all possible harmful sources, were designed-the detection probability and the saturation time. They were evaluated for several meteorological situations and emission characteristics. The detection probability proves to be a reliable indicator, whereas the saturation time is too dependent on the dispersion conditions and the emitted quantity. Although it provides some measure of the behavior with respect to time, other indicators should also be tested, such as the averaged value of the arrival times on all sources (instead of the maximum value). In cases when the time of response is critical, one may also impose a minimum time between the source detection and the actual contamination 
of $D_{p}$. This study also highlights the need for appropriate sizes for the simulation domain, so as to ensure that all effective sources are taken into account for a given emitted quantity and meteorological situation. However, the networks should accommodate meteorological variations (wind speed and direction, rain) without a total loss of performance, which ensures that a few well-chosen meteorological situations should be representative of a network protection ability. Finally, two methods to design an efficient network were evaluated: (1) taking the best sensors according to the detection probability, and (2) placing the sensors into circles around the protected domain. Placing the best sensors according to the saturation time gave poor networks performance, and was therefore not further investigated. While the first method depends on the wind direction, which makes it more suitable for the units protection scenario (real-time sensors siting), the second gives good results for the protection of larger areas containing buildings. Imposing a sufficient scattering of the sensors is a critical condition to ensure complementarity between the sensors and thus good performance for a reasonable number of sensors. This can be achieved by means of indicators coupling (e.g., choosing the sensors with constraints on both the detection probability and the saturation time), by imposing a minimum distance between two sensors, or by discarding any source already detected by a network when computing the statistics of a sensor to be included. A good scattering is also ensured by designing the network with concentric circles, if enough sensors are available.

The next step of such a study could be a network optimization that would maximize a statistical indicator on all sources and meteorological situations. Another perspective is the optimization of networks for the characterization of a source (place, date and quantity) through inversion methods. Finally, it would also be useful to extend this study to the prediction of the zones contaminated by a given source.

\section{Acknowledgements}

This study was conducted for DGA (Délégation Générale de l'Armement- http://www . defense.gouv.fr/dga/) and is published with its authorization. Polyphemus has been developed at CEREA (Joint research laboratory ENPC - EDF R\&D, Université Paris-Est) http://www. cerea.enpc.fr/) and INRIA (CLIME project- http://www-rocq.inria. $\mathrm{fr} / \mathrm{clime}$ ). It has been financially supported by IRSN (http://www.irsn.fr) and DGA.

\section{References}

[1] Abida, R., Bocquet, M., Vercauteren, N., Isnard, O., 2008. Design of a monitoring network over france in case of a radiological accidental release. Atmos. Env. 42, 5205-5219.

[2] Belot, Y., Caput, C., Guenot, J., 1988. Etude bibliographique du lavage par la pluie des radionucléides particulaires et gazeux émis en situation accidentelle. Tech. rep., IRSN et EDF.

[3] Boutahar, J., Lacour, S., Mallet, V., Quélo, D., Roustan, Y., Sportisse, B., 2004. Development and validation of a fully modular platform for numerical modelling of air pollution: POLAIR. Int. J. Env. and Pollution 22 (1/2), 17-28.

[4] Fuentes, M., Chaudhuri, A., Holland, D. M., 2007. Bayesian entropy for spatial sampling design of environmental data. Environ. Ecol. Stat. 14, 323-340.

[5] Jiang, Z., de Bruin, S., Heuvelink, G., Twenhöfel, C., 2007. Optimization of mobile radioactivity monitoring networks. In: Fifth International Symposium on Spatial Data Quality.

[6] Korsakissok, I., Mallet, V., 2008. Comparative study of Gaussian dispersion formulae within the Polyphemus platform: evaluation with Prairie Grass and Kincaid experiments. J. Applied Meteor.Submitted.

[7] Krysta, M., Bocquet, M., Sportisse, B., Isnard, O., 2006. Data assimilation for short-range dispersion of radionuclides: An application to wind tunnel data. Atmos. Env. 40, 7267-7279. 
[8] Mallet, V., Quélo, D., Sportisse, B., Ahmed de Biasi, M., Debry, É., Korsakissok, I., Wu, L., Roustan, Y., Sartelet, K., Tombette, M., Foudhil, H., 2007. Technical Note: The air quality modeling system Polyphemus. Atmos. Chem. Phys. 7 (20), 5479-5487.

[9] Nychka, D., Saltzman, N., 1998. Design of air quality monitoring networks. In: Nychka, D., Cox, L., Piegorsch, W. (Eds.), Case Studies in Environmental Statistics. Lecture Notes in Statistics 120. Springer-Verlag, pp. 51-76.

[10] Ramaker, J., 2003. The final test a history of the comprehensive nucelar-test-ban treaty negotiations. Tech. rep., Provisional Technical Secretariat of the Preparatory Commission for the CTBTO.

[11] Underwood, B., 2001. Review of deposition velocity and washout coefficient. Tech. rep., AEA Technology, Harwell.

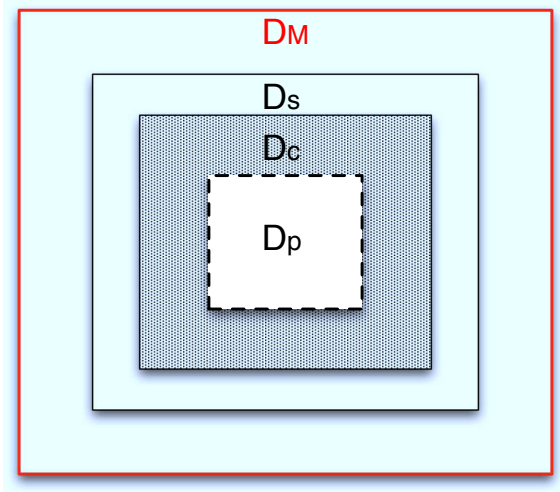

Figure 1: Domains: $D_{p}$ is the zone to be protected, $D_{c}$ is the domain of sensors, $D_{s}$ is the domain of sources, and $D_{M}$ is the modeled domain. 


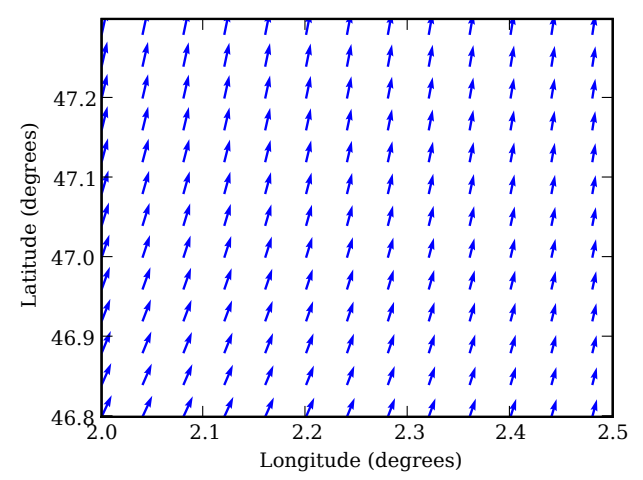

(a) Case 0

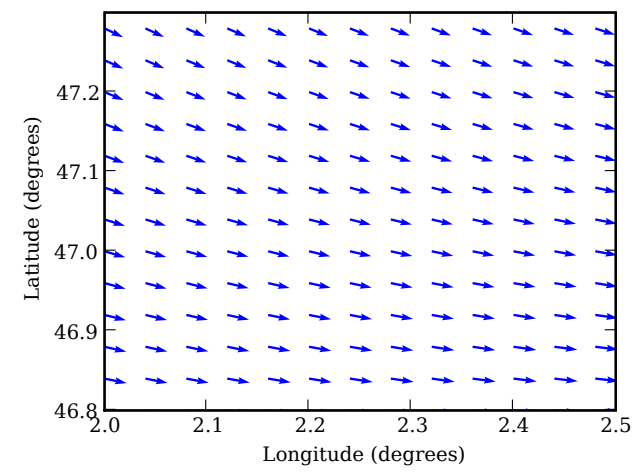

(c) Case 2

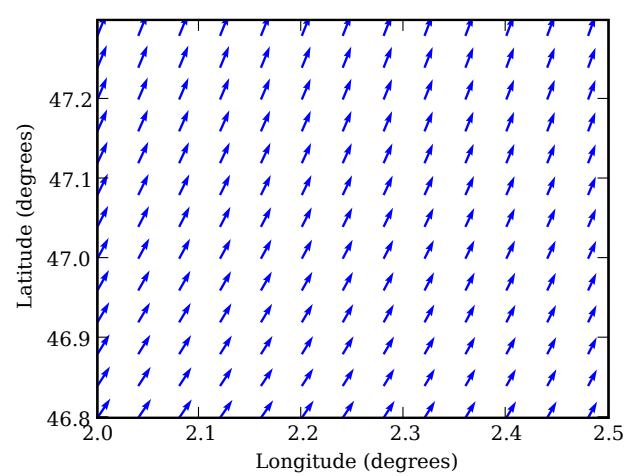

(b) Case 1

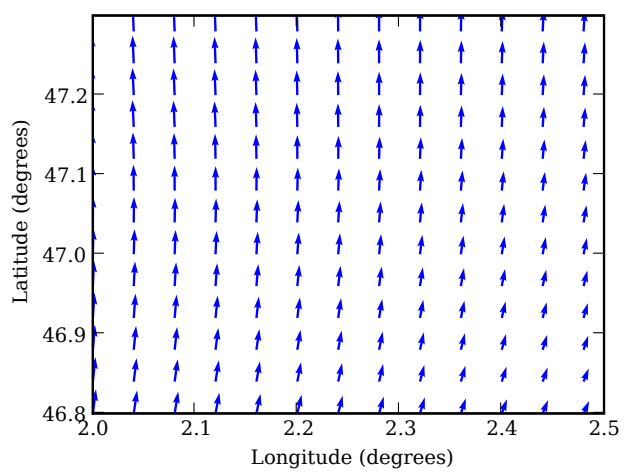

(d) Case 3

Figure 2: Map of winds at the emission time for each meteorological situation at regional scale. Note that only wind direction is represented, the arrow lengths are not proportional to the speed. 


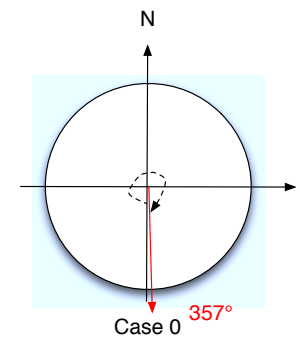

$\mathrm{N}$

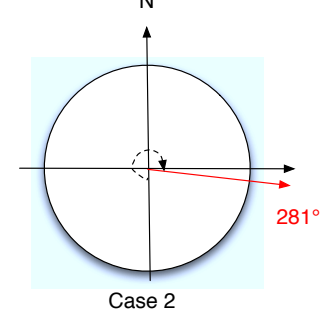

Case 2

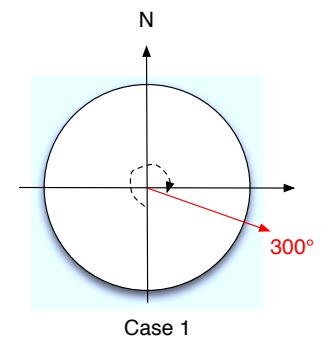

$\mathrm{N}$

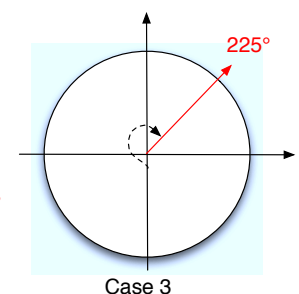

Figure 3: Wind direction for each meteorological situation at local scale. The direction of the wind $u$ is shown, and the wind angle from north is indicated in degrees. 


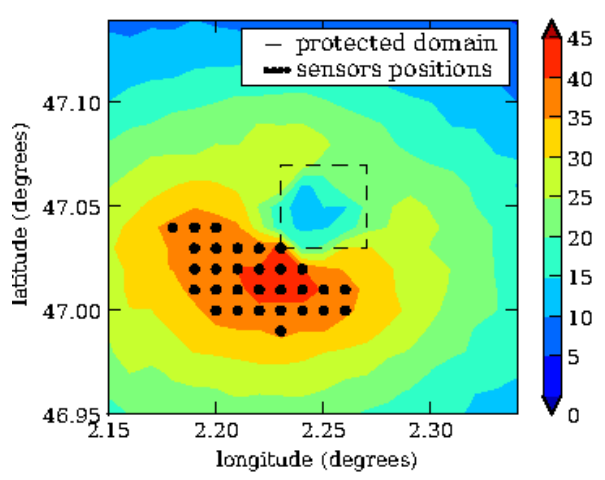

(a) Situation 0

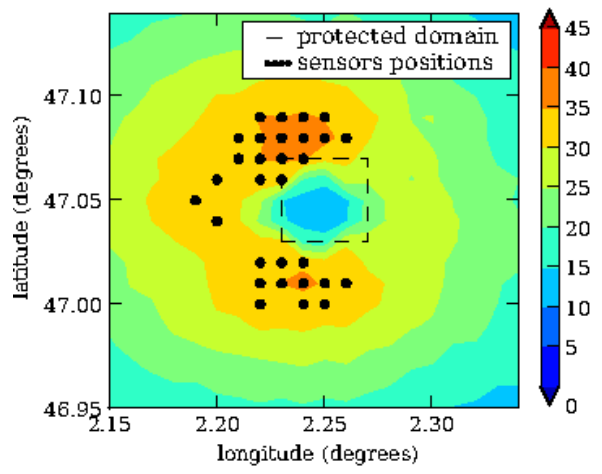

(c) Situation 2

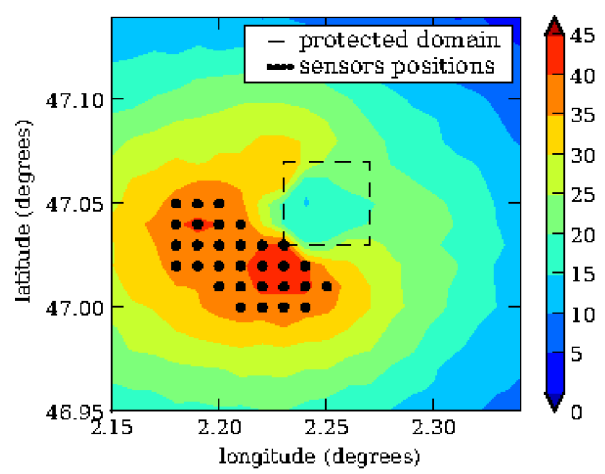

(b) Situation 1

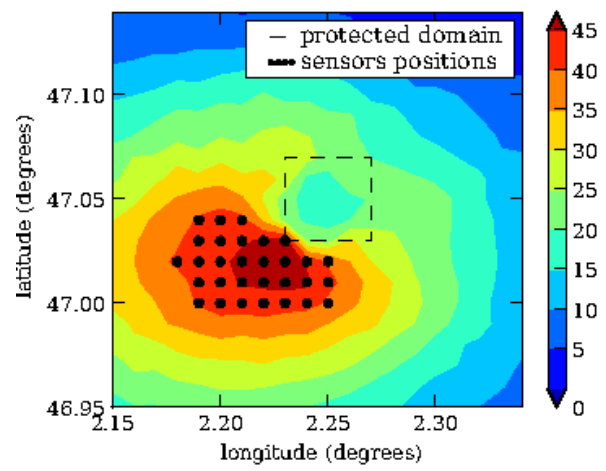

(d) Situation 3

Figure 4: Regional scale: Map of maximum detection probability (percent) for all sensors in the domain $D_{c}$ and the four meteorological situations. The black dots are the positions of the 30 sensors having the highest detection probability. 


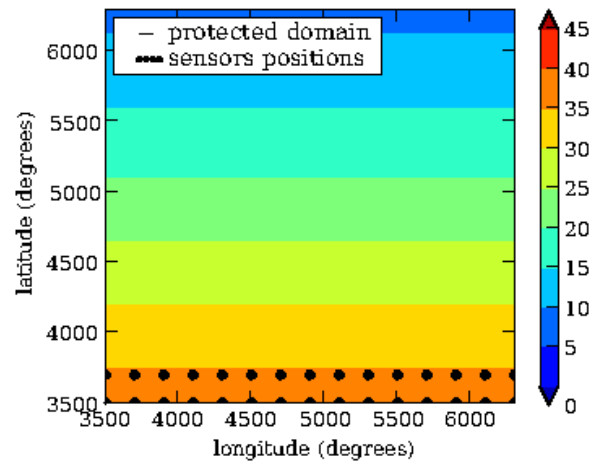

(a) Situation 0

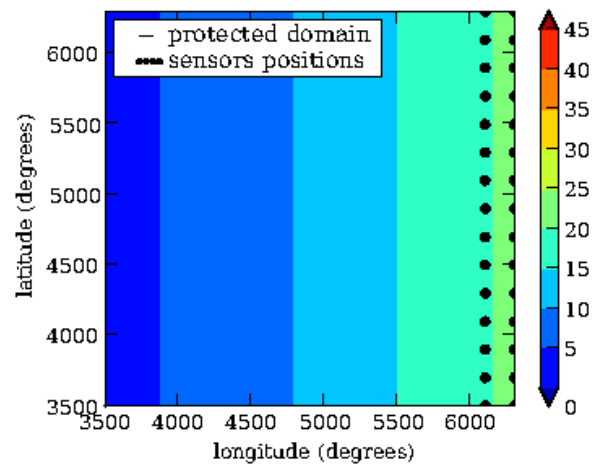

(c) Situation 2

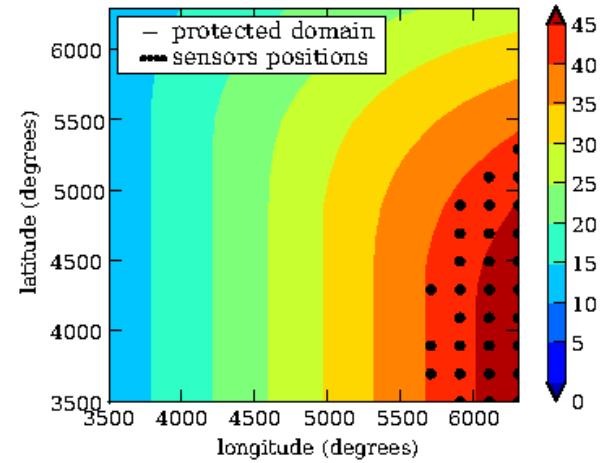

(b) Situation 1

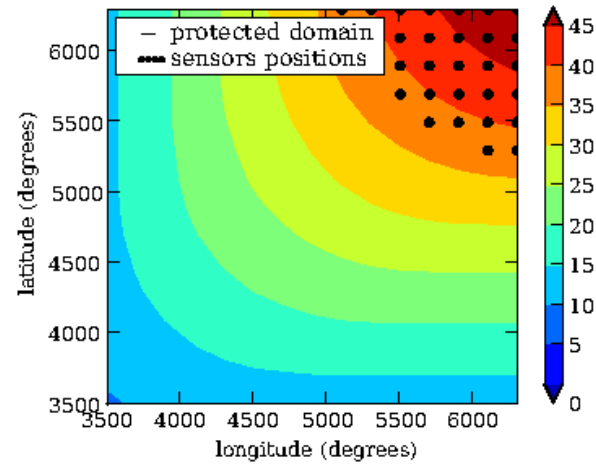

(d) Situation 3

Figure 5: Local scale: Map of maximum detection probability (percent) for all sensors in the domain $D_{c}$ and the four meteorological situations. The black dots are the positions of the 30 sensors having the highest detection probability. The dashed line is the boundary of $D_{p}$. 


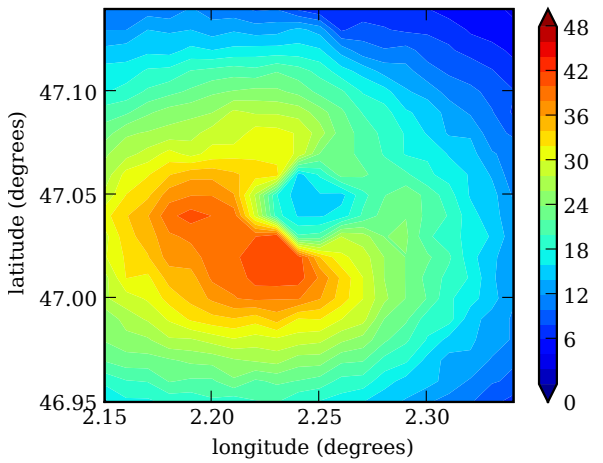

(a) $P_{\max }$ for iodine

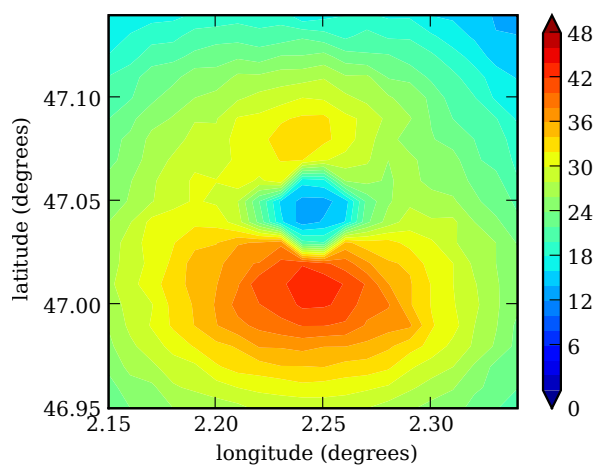

(c) $P_{\max }$ for GM

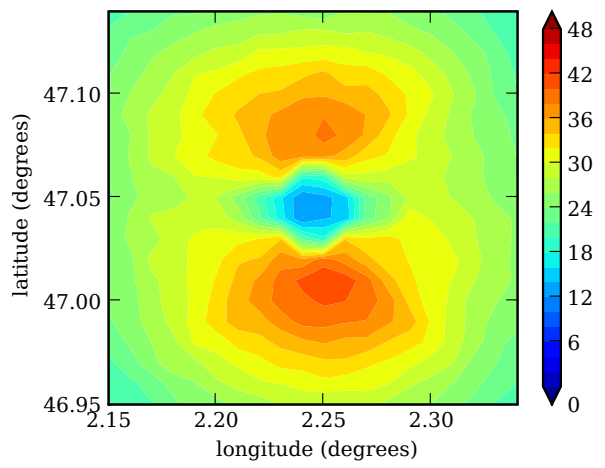

(e) $P_{\max }$ for biological PM - diameter $0.5 \mu \mathrm{m}$

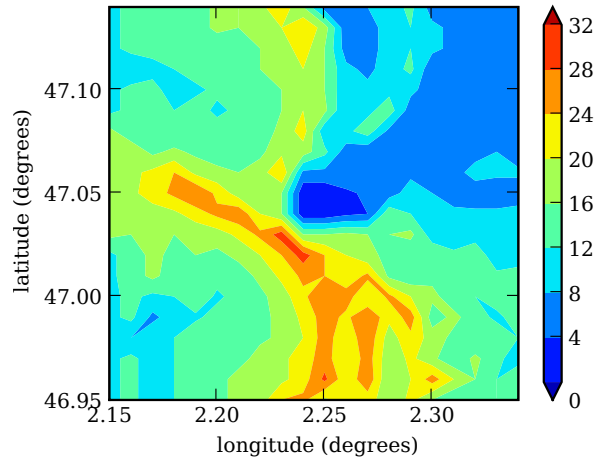

(b) $t_{\text {sat }}$ for iodine

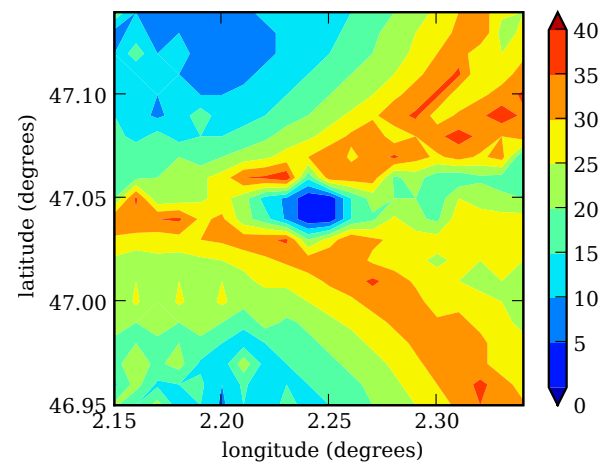

(d) $t_{\text {sat }}$ for GM

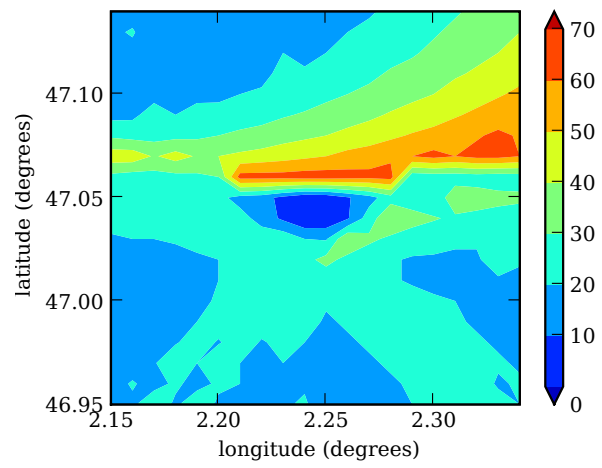

(f) $t_{\text {sat }}$ for biological PM - diameter $0.5 \mu \mathrm{m}$

Figure 6: Maps of maximum detection probability (in \%) and saturation time (in minutes) for all sensors in the domain $D_{c}$ and three different species - Meteorological situation 1 (low dispersion, winter, daytime). The emitted quantities are: $1 \mathrm{~kg}$ of iodine, $5 \mathrm{~kg}$ of GM and $10 \mathrm{~kg}$ of biological tracer for each diameter. The dashed line is the boundary of $D_{p}$. 


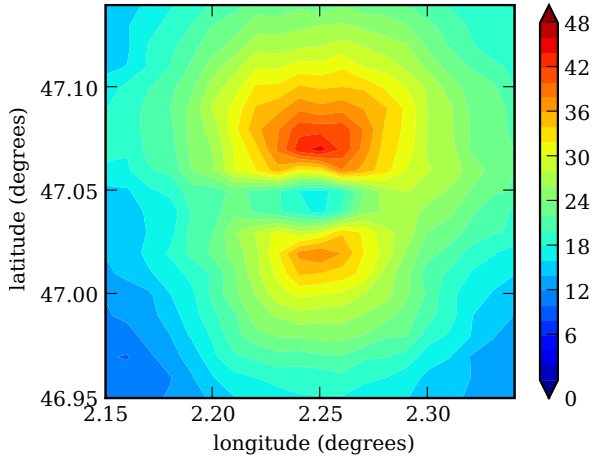

(a) Biological PM - diameter $0.5 \mu \mathrm{m}$

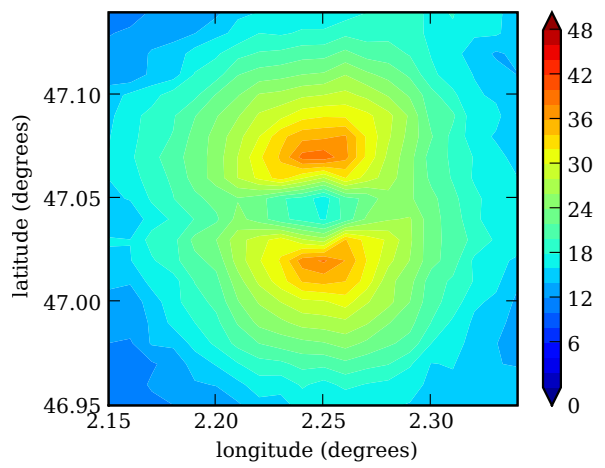

(c) Biological PM - diameter $10 \mu \mathrm{m}$

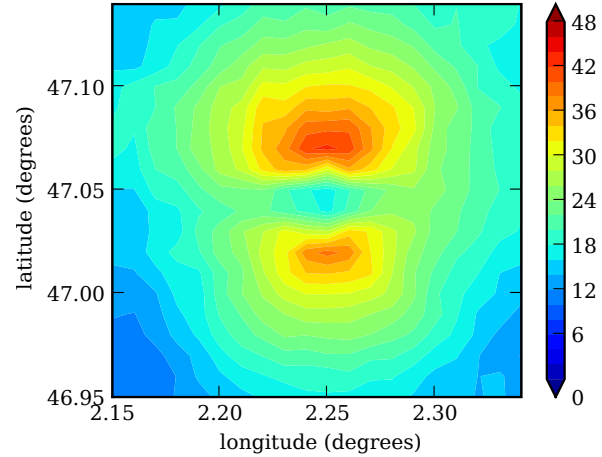

(b) Biological PM - diameter $5 \mu \mathrm{m}$

Figure 7: Map of maximum detection probability (percent) for all sensors in the domain $D_{c}$ and biological particle of three different diameters - Meteorological situation 2 (low dispersion, summer, nighttime, rain). The dashed line is the boundary of $D_{p}$. 


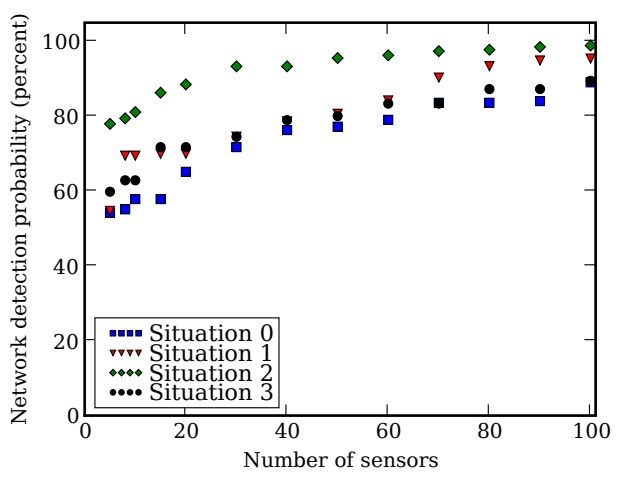

(a) $P_{\max }$, regional scale

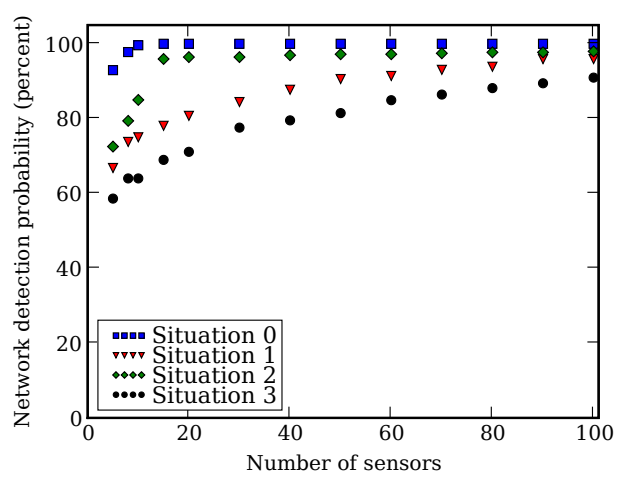

(c) $P_{\text {max }}$, local scale

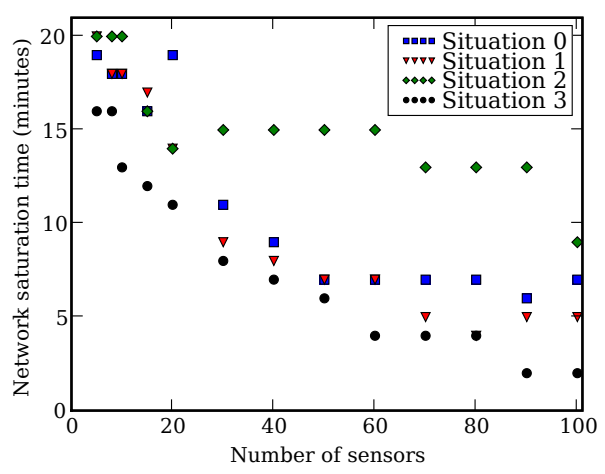

(b) $t_{\text {sat }}$, regional scale

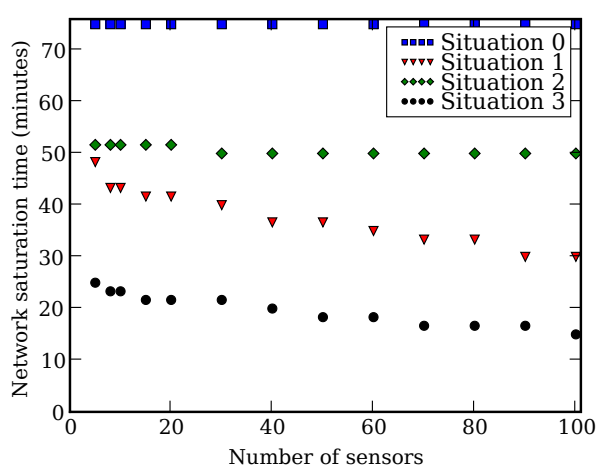

(d) $t_{\text {sat }}$, local scale

Figure 8: Performance of the networks defined with the sensors having the highest detection probabilities against the number of sensors, for all meteorological situations, at local and regional scale. The species is iodine.
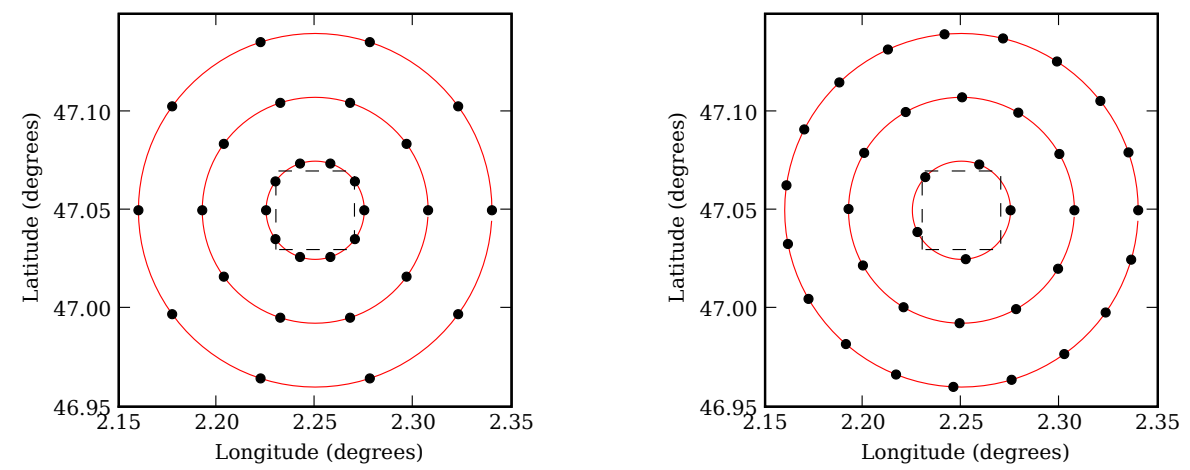

(a) Sensors position for 10 sensors per circle (b) Sensors position for a constant distance between $\left(N_{\text {sensor }}^{0}=10.\right)$ 2 sensors equal to $3 \mathrm{~km}$.

Figure 9: Sensors positions for two network configurations with 3 concentric circles of radii $2.5 \mathrm{~km}, 5.75 \mathrm{~km}$ and $9 \mathrm{~km}$. The dashed lines represent the boundaries of the protected domain $D_{p}$. 

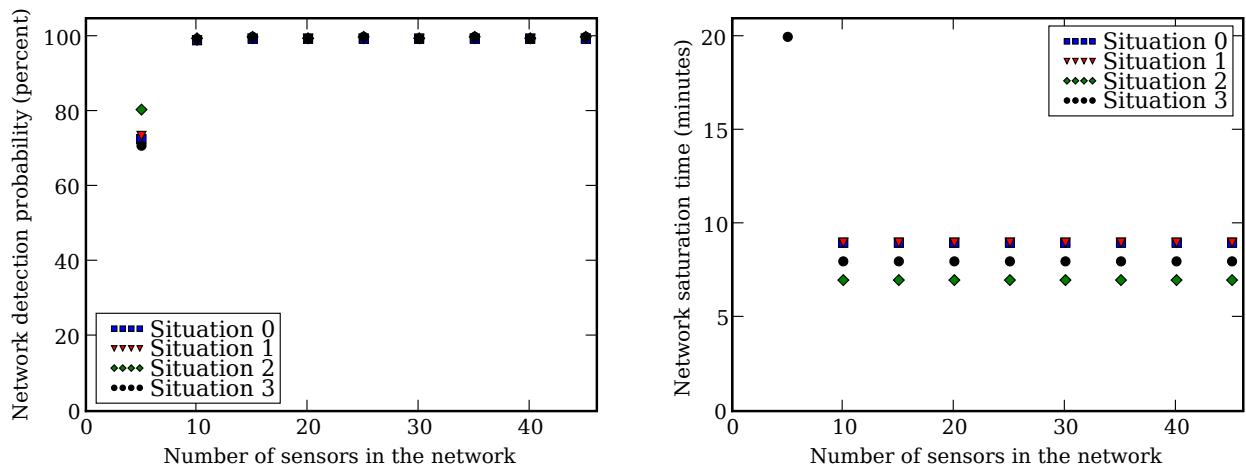

(a) $P_{\max }$ against $N_{\text {sensor }}=5 \times N_{\text {circle }}$. $N_{\text {circle }}$ varies be- (b) $t_{\text {sat }}$ against $N_{\text {sensor }}=5 \times N_{\text {circle }}$. $N_{\text {circle }}$ varies between tween 1 and 9 . 1 and 9 .
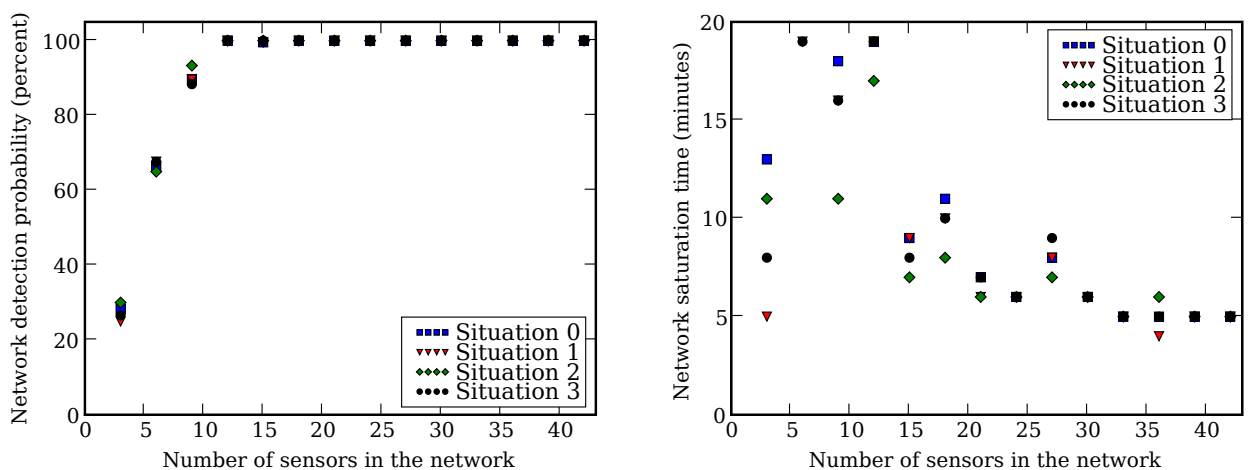

(c) $P_{\text {max }}$ against $N_{\text {sensor }}=3 \times N_{\text {sensor }}^{0}$. $N_{\text {sensor }}^{0}$ varies be- (d) $t_{\text {sat }}$ against $N_{\text {sensor }}=3 \times N_{\text {sensor }}^{0} . N_{\text {sensor }}^{0}$ varies between 1 and 14 . tween 1 and 14
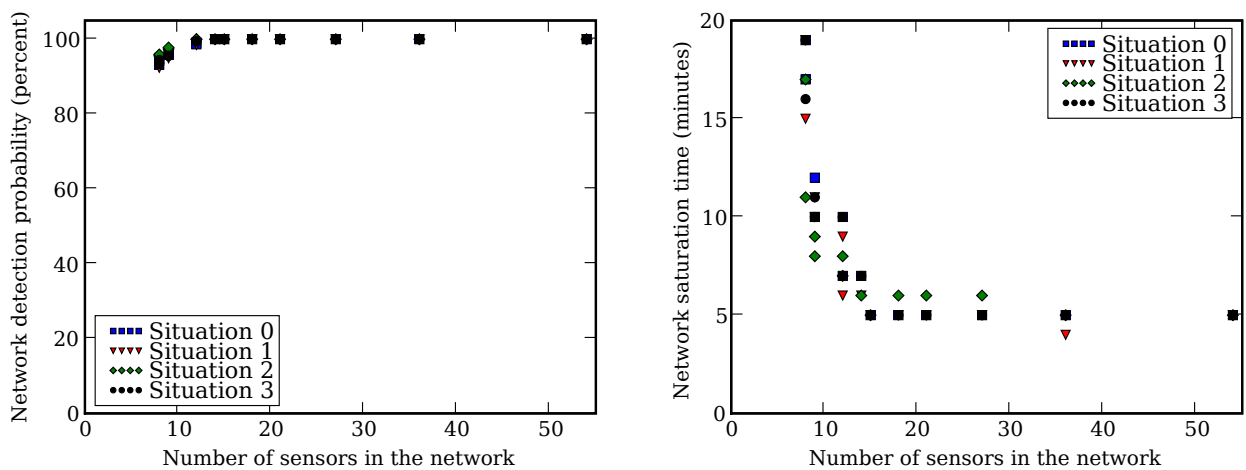

(e) $P_{\max }$ against $N_{\text {sensor }}$ given by Eq. $13\left(N_{\text {circle }}=3\right)$. (f) $t_{\text {sat }}$ against $N_{\text {sensor }}$ given by Eq. $13\left(N_{\text {circle }}=3\right)$. $l_{\text {sensor }}$ $l_{\text {sensor }}$ varies between 2 and $15 \mathrm{~km}$. varies between 2 and $15 \mathrm{~km}$.

Figure 10: Networks detection probability and saturation time with respect to $N_{\text {sensor }}$ for several configurations in circles. The circles radii are comprised between $2.5 \mathrm{~km}$ and $9 \mathrm{~km}$. In 10(a) and 10(b), the number of circles varies, for 5 sensors per circle. In 10 (c) and 10(d), the number of sensors per circle varies, for 3 circles. In 10(e) and 10(f), the distance between two sensors $l_{\text {sensor }}$ varies, for 3 circles. 


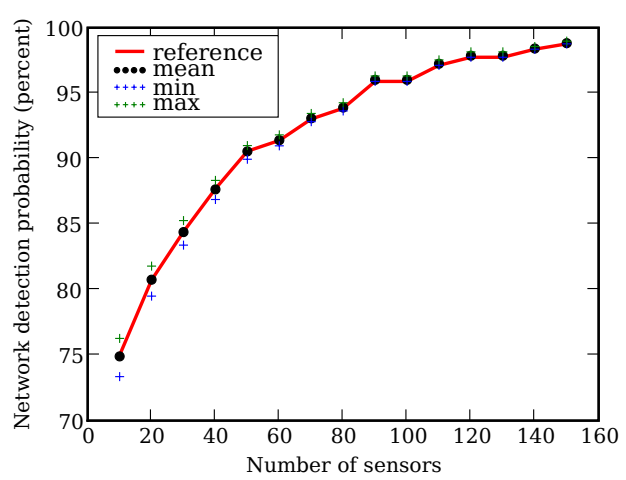

(a) Network detection probability - local scale

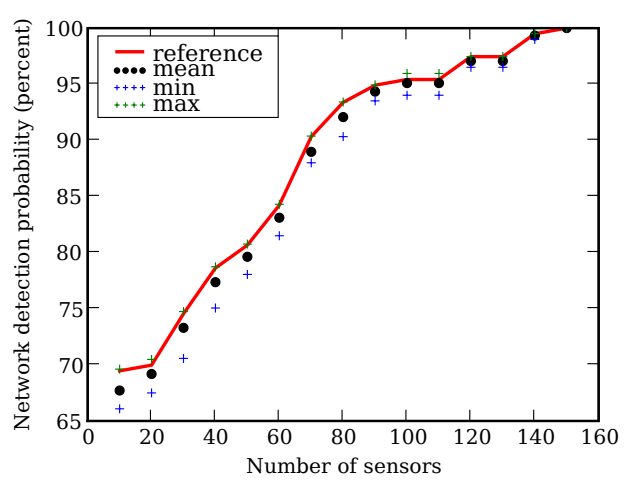

(c) Network detection probability - regional scale

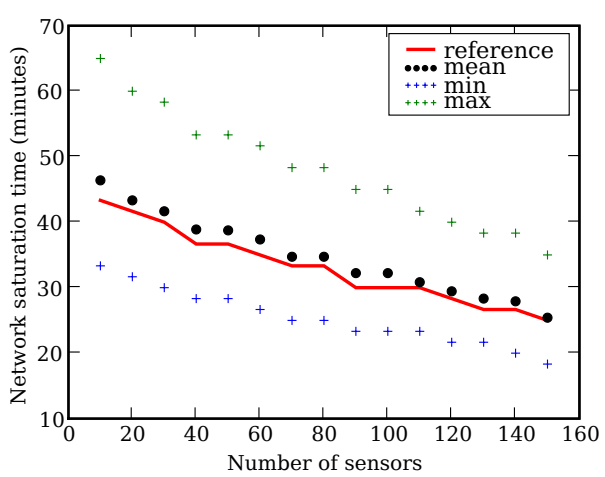

(b) Network saturation time - local scale

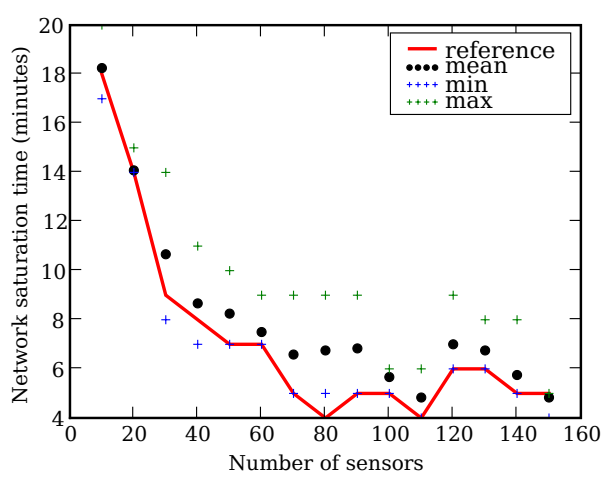

(d) Network saturation time - regional scale

Figure 11: Influence of wind speed variations: evolution of the mean detection probability and saturation time with a wind speed variation between $-35 \%$ and $+35 \%$, at local and regional scale. Reference is the situation 1 . Networks are defined with the sensors having the highest detection probability. 


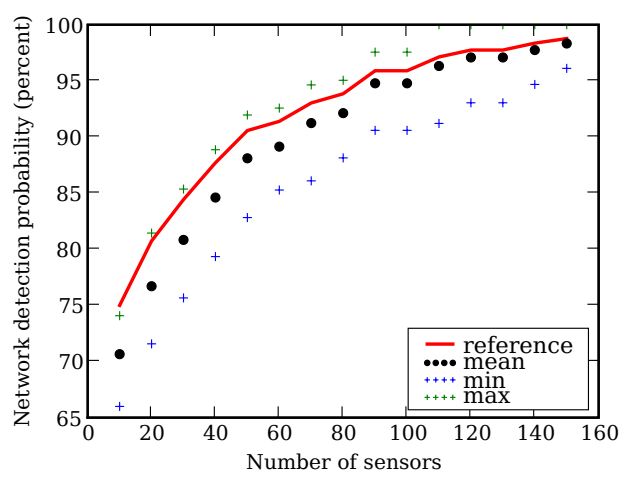

(a) Network detection probability - local scale

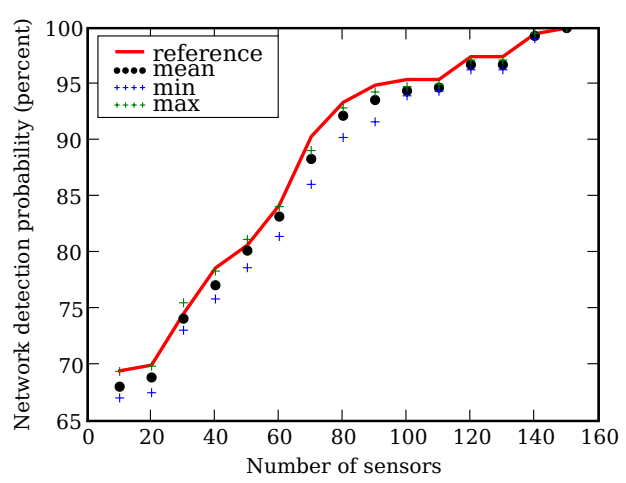

(c) Network detection probability - regional scale

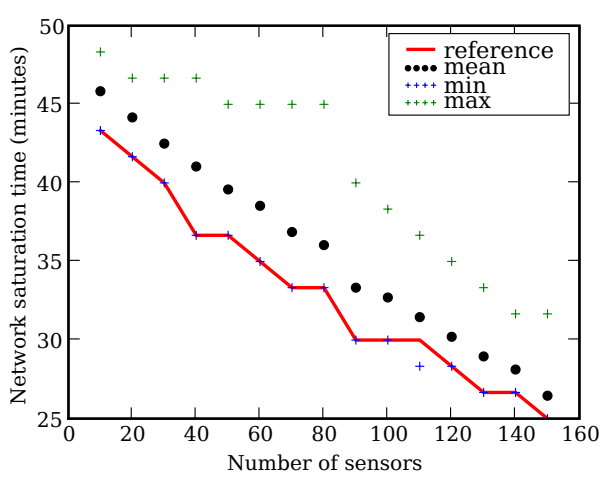

(b) Network saturation time - local scale

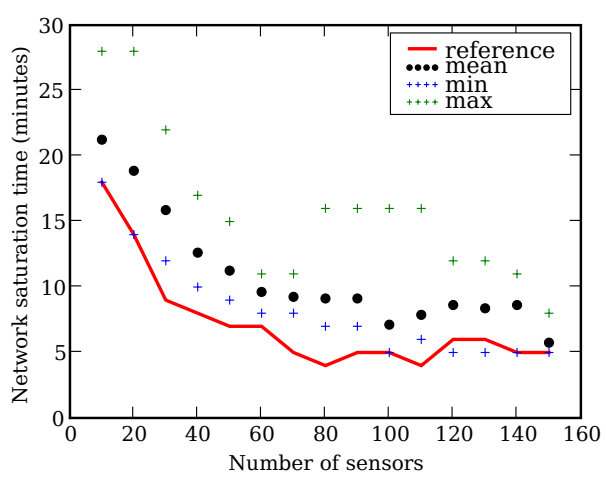

(d) Network saturation time - regional scale

Figure 12: Influence of wind angle variations: variations of detection probability and saturation time with a wind speed variation between $-25^{\circ}$ and $+25^{\circ}$ at local and regional scale. Reference is the situation 1 . Networks are defined with the sensors having the highest detection probability. 


\begin{tabular}{cc}
\hline Domain & Dimension \\
\hline$D_{p}$ & $4 \times 4 \mathrm{~km}^{2}$ \\
$D_{c}$ & $20 \times 20 \mathrm{~km}^{2}$ \\
$D_{s}$ & $31 \times 31 \mathrm{~km}^{2}$ \\
$D_{M}$ & $50 \times 50 \mathrm{~km}^{2}$ \\
\hline
\end{tabular}

Table 1: Size of the different domains at regional scale.

\begin{tabular}{cc}
\hline Domain & Dimension \\
\hline$D_{p}$ & $3 \times 3 \mathrm{~km}^{2}$ \\
$D_{c}$ & $3 \times 3 \mathrm{~km}^{2}$ \\
$D_{s}$ & $6.2 \times 6.2 \mathrm{~km}^{2}$ \\
$D_{M}$ & $10 \times 10 \mathrm{~km}^{2}$ \\
\hline
\end{tabular}

Table 2: Size of the different domains at local scale.

\begin{tabular}{ccc}
\hline Species & Diameter $(\mu \mathrm{m})$ & Quantity $(\mathrm{kg})$ \\
\hline Iodine & gas & 1. \\
GM & gas & 5. \\
Biological & 0.5 & 10. \\
Biological & 5. & 10. \\
Biological & 10. & 10. \\
\hline
\end{tabular}

Table 3: Emitted quantities for the different species. The quantities are emitted instantaneously. 


\begin{tabular}{cccccc}
\hline Date & Mean wind & Mean BLH & Mean rain & Cloudiness & Stability \\
\hline 2004-01-04 09h & 1.05 & 175 & 0. & 0.47 & B \\
2004-01-04 15h & 1.59 & 250 & 0.01 & 0.46 & B \\
2004-08-10 03h & 1.66 & 308 & 3.89 & 0.99 & F \\
2004-08-23 09h & 2.97 & 565 & 0.11 & 0.59 & C \\
\hline
\end{tabular}

Table 4: Wind speed in $\mathrm{m} \mathrm{s}^{-1}$, boundary layer height (BLH) in $m$, rain intensity in $\mathrm{mm} \mathrm{hr}^{-1}$, cloudiness $(0$ is clear sky, 1 is a total cloudiness) and Pasquill stability class for the four meteorological situations at regional scale (averaged values over the domain and simulation duration). The hours given here are local time.

\begin{tabular}{ccccc}
\hline Case & Wind & Boundary height & Rain & Stability \\
\hline 0 & 1.01 & 152 & 0. & B \\
1 & 1.66 & 244 & 0.003 & B \\
2 & 1.42 & 300 & 4.19 & F \\
3 & 3.46 & 623 & 0.18 & C \\
\hline
\end{tabular}

Table 5: Wind speed in $\mathrm{m} \mathrm{s}^{-1}$, boundary layer height in $m$, rain intensity in $\mathrm{mm} \mathrm{hr}^{-1}$ and Pasquill stability class for the four meteorological situations at local scale. 